\title{
ON INVERTIBLE OPERATORS AND INVARIANT SUBSPACES
}

\author{
AVRAHAM FEINTUCH ${ }^{1}$
}

\begin{abstract}
Let $A$ be an invertible operator on a complex Hilbert space $H$. Sufficient conditions are given for the inverse of $A$ to be a weak limit of polynomials in $A$.
\end{abstract}

1. Introduction. Let $H$ be a complex Hilbert space. If $H$ is finite dimensional and $A$ is an invertible linear operator on $H$, then there is a polynomial $p$ such that $A^{-1}=p(A)$. The infinite-dimensional analogue of this fact is generally false. If $U$ is any unitary operator which contains a bilateral shift direct summand, then $U^{-1}=U^{*}$ is not a weak limit of polynomials in $U$ [4]. In this paper two sufficient conditions, quite different in nature, are given for the inverse of a bounded linear operator to be a weak limit of polynomials in the operator.

2. Preliminaries. If $A$ is a bounded linear operator on $A$, then Lat $A$ represents the lattice of closed invariant subspaces of $A . H^{(n)}$ will denote the usual orthogonal direct sum of $n$ copies of $H$. A typical vector in $H^{(n)}$ will be denoted by $\left\langle x_{1}, \cdots, x_{n}\right\rangle$ with $x_{i} \in H$. If $A$ is an operator on $H$, let $A^{(n)}$ denote the operator $\sum_{i=1}^{n} \oplus A_{i}$ on $H^{(n)}$ with $A_{i}=A$ for all $i$. The inner product on $H$ will be denoted by ( , ).

The following lemma is a special case of a well-known result [3].

Lemma 1. Let $A$ be a bounded invertible operator on $H$. If Lat $A^{(n)} \subseteq$ Lat $A^{-1(n)}$ for all integers $n \geqq 1$, then $A^{-1}$ is a weak limit of polynomials in $A$.

\section{Numerical range.}

Definition. Let $A$ be a bounded operator on $H$. The numerical range of $A$ is the set $\omega(A)=\{(A x, x):\|x\|=1\}$.

Lemma 2. If $A \in B(H)$, then $\omega(A)=\omega\left(A^{(n)}\right)$ for all integers $n \geqq 1$.

Received by the editors December 1, 1972 and, in revised form, June 8, 1973.

AMS (MOS) subject classifications (1970). Primary 47B10.

Key words and phrases. Invertible operator, invariant subspace, numerical range, operator range.

1 This paper is part of the author's dissertation at the University of Toronto. The author would like to thank Professor Peter Rosenthal for many valuable discussions which led to the results of this paper.

(c) American Mathematical Society 1974 
Proof. Suppose $t \in \omega(A)$. Then there is a unit vector $x$ in $H$ such that $(A x, x)=t$. A consideration of $\left(A^{(n)} y, y\right)$, for $y=\langle x, 0, \cdots, 0\rangle$, gives $t \in \omega\left(A^{(n)}\right)$. Thus $\omega(A) \subset \omega\left(A^{(n)}\right)$.

Now suppose $t$ is in $\omega\left(A^{(n)}\right)$. Thus there exist vectors $x_{1}, \cdots, x_{n}$ in $H$ with $\sum_{i=1}^{n}\left\|x_{i}\right\|^{2}=1$ such that $\sum_{i=1}^{n}\left(A x_{i}, x_{i}\right)=t$. Now $1 /\left\|x_{i}\right\|^{2}\left(A x_{i}, x_{i}\right) \in$ $\omega(A)$ for $1 \leqq i \leqq n$. Since $\omega(A)$ is convex, it follows that

$$
\sum_{i=1}^{n}\left\|x_{i}\right\|^{2} \frac{1}{\left\|x_{i}\right\|^{2}}\left(A x_{i}, x_{i}\right)=\sum_{i=1}^{n}\left(A x_{i}, x_{i}\right)=t
$$

is in $\omega(A)$. This completes the proof.

Lemma 3. Let $A$ be a bounded invertible operator on $H$. Then $0 \notin \omega(A)$ implies Lat $A \subset$ Lat $A^{-1}$.

Proof. Suppose there is $M \in$ Lat $A$ which is not invariant under $A^{-1}$. Since $A$ is invertible, it follows that $A M$ is a closed subspace of $M$. Let $N=M \ominus A M$.

Let $x$ be a unit vector in $N$. $N \subset M$ implies $A x \in A M$ and thus $(A x, x)=0$. Since $0 \notin \omega(A), N$ must be zero.

Notation. The weak closure of the algebra of polynomials in $A$ will be denoted by $U_{A}$.

THEOREM 1. If $A$ is an invertible operator on $H$ and $0 \notin \omega(A)$, then $U_{A}=U_{A^{-1}}$.

Proof. $0 \notin \omega(A)$ implies $0 \notin \omega\left(A^{-1}\right)$. For, if there exists $f \in H$ such that $\left(A^{-1} f, f\right)=0$, then since $A$ is invertible, $f=A g, g \in H$. Thus

$$
0=\left(A^{-1} f, f\right)=\left(A^{-1} A g, A g\right)=(g, A g)=\left(A^{*} g, g\right) .
$$

By normalizing, if necessary, and using the fact that $\omega\left(A^{*}\right)=(\omega(A))^{*}$ we obtain $0 \in \omega(A)$.

Thus the result will be symmetric in $A$ and $A^{-1}$. The fact that Lat $A^{(n)}=$ Lat $A^{-1(n)}$ follows immediately from Lemmas 2 and 3 . This completes the proof.

\section{Operator ranges.}

Definition. A linear manifold $L \subset H$ is an operator range if there exists a Hilbert space $K$ and a bounded operator $A$ from $K$ to $H$ such that $L=A K$. The idea of studying the invariant operator ranges of an algebra of operators was introduced by Foias [2] and the basic facts about operator ranges can be found in an excellent account by Fillmore and Williams [1].

If $A$ is a bounded invertible operator on $H$, then a necessary condition for $A^{-1} \in U_{A}$ is Lat $A \subset$ Lat $A^{-1}$. It is not known if this is also sufficient. 
However, if every invariant linear manifold of $A$ is invariant under $A^{-1}$, it follows from a result of $\mathrm{P}$. Fillmore that $A^{-1}=p(A)$ for some polynomial $p$. Here we present what could be considered the intermediate result. The lattice of invariant operator ranges of $A$ will be denoted by $\mathrm{Lat}_{1 / 2} A$.

TheOREM 2. Let $A$ be an invertible operator on $H$. Then Lat $_{1 / 2} A \subset$ Lat $_{1 / 2} A^{-1}$ implies $A^{-1} \in U_{A}$.

Proof. We show, by induction, that Lat $A^{(n)} \subseteq$ Lat $A^{-1(n)}$. By hypothesis, it is true for $n=1$ so assume Lat $A^{(i)} \subset$ Lat $A^{-1(i)}$ for $i<n$ and suppose $M \in$ Lat $A^{(n)}$. We consider two cases.

Case (1). $M$ does not contain a vector of the form $\left\langle 0, y_{1}, \cdots, y_{n-1}\right\rangle$ other than the zero vector. Then the first component uniquely determines every other component and, since $M$ is a linear space, this determination is linear. Thus there exist (possibly unbounded) linear transformations $T_{1}, \cdots, T_{n-1}$ such that

where

$$
M=\left\{\left\langle x, T_{1} x, \cdots, T_{n-1} x\right\rangle: x \in D\right\}
$$

$$
D=\left\{x: \exists x_{1}, \cdots, x_{n-1} \text { with }\left\langle x, x_{1}, \cdots, x_{n-1}\right\rangle \in M\right\} .
$$

Since $M$ is closed and $D$ is the range of the projection onto the first coordinate space of $M, D$ is an operator range. $M \in$ Lat $A^{(n)}$ implies $D \in \mathrm{Lat}_{1 / 2} A \subset \mathrm{Lat}_{1 / 2} A^{-1}$.

Now $M \in$ Lat $A^{(n)}$ implies $A T_{i}=T_{i} A$ for $1 \leqq i \leqq n-1$. Thus

$$
A^{-1} T_{i}=A^{-1} T_{i} A A^{-1}=A^{-1} A T_{i} A^{-1}=T_{i} A^{-1} .
$$

Thus $M \in$ Lat $A^{-1(n)}$. Let

Case (2). Assume $M$ contains a nontrivial vector $\left\langle 0, y_{1}, \cdots, y_{n-1}\right\rangle$.

$$
N=\left\{\left\langle 0, y_{1}, \cdots, y_{n-1}\right\rangle \in M\right\} .
$$

By the induction hypothesis $N \in$ Lat $A^{-1(n)}$ :

Let $M^{\prime}=M \ominus N$. The argument used in Case (1) shows that $M^{\prime}$ is of the form $\left\{\left\langle x, T_{1} x, \cdots, T_{n-1} x\right\rangle: x \in D\right\}$ with $D \in \mathrm{Lat}_{1 / 2} A \subset \mathrm{Lat}_{1 / 2} A^{-1}$. If $\left\langle x, T_{1} x, \cdots, T_{n-1} x\right\rangle \in M^{\prime}$, then

$$
\begin{aligned}
A^{(n)}\left\langle x, T_{1} x, \cdots, T_{n-1} x\right\rangle= & \left\langle A x, T_{1} A x, \cdots, T_{n-1} A x\right\rangle \\
& +\left\langle 0,\left(A T_{1}-T_{1} A\right) x, \cdots,\left(A T_{n-1}-T_{n-1} A\right) x\right\rangle,
\end{aligned}
$$

where the first term is in $M^{\prime}$ and the second in $N$. Since $N \in$ Lat $A^{-1(n)}$,

$$
\begin{aligned}
A^{-1(n)}\left\langle 0,\left(A T_{1}-\right.\right. & \left.\left.T_{1} A\right) x, \cdots,\left(A T_{n-1}-T_{n-1} A\right) x\right\rangle \\
& =\left\langle 0, T_{1} x, \cdots, T_{n-1} x\right\rangle-\left\langle 0, A^{-1} T_{1} A x, \cdots, A^{-1} T_{n-1} A x\right\rangle
\end{aligned}
$$


is in $N$. Let $Q$ be the projection on $N^{\perp}$ in $H^{(n)}$. Then since $\left\langle 0, T_{1} x, \cdots\right.$, $\left.T_{n-1} x\right\rangle \in N^{\perp}$,

$$
\left\langle 0, T_{1} x, \cdots, T_{n-1} x\right\rangle=Q\left\langle 0, A^{-1} T_{1} A x, \cdots, A^{-1} T_{n-1} A x\right\rangle .
$$

We must show that $A^{-1(n)}\left\langle x, T_{1} x, \cdots, T_{n-1} x\right\rangle \in M$. Since $A D=D$, there is some $y \in D$ such that $x=A y$. Thus $\left\langle x, T_{1} x, \cdots, T_{n-1} x\right\rangle=$ $\left\langle A y, T_{1} A y, \cdots, T_{n-1} A y\right\rangle$. Then

$$
\begin{aligned}
& A^{-1(n)}\left\langle x, T_{1} x, \cdots, T_{n-1} x\right\rangle=\left\langle y, A^{-1} T_{1} A y, \cdots, A^{-1} T_{n-1} A y\right\rangle \\
&=\langle y, 0, \cdots, 0\rangle+\left\langle 0, A^{-1} T_{1} A y, \cdots, A^{-1} T_{n-1} A y\right\rangle \\
&=\langle y, 0, \cdots, 0\rangle+Q\left\langle 0, A^{-1} T_{1} A y, \cdots, A^{-1} T_{n-1} A y\right\rangle \\
&+\left(I^{(n)}-Q\right)\left\langle 0, A^{-1} T_{1} A y, \cdots, A^{-1} T_{n-1} A y\right\rangle \\
&=\langle y, 0, \cdots, 0\rangle+\left\langle 0, T_{1} y, \cdots, T_{n-1} y\right\rangle \\
&+\left(I^{(n)}-Q\right)\left\langle 0, A^{-1} T_{1} A y, \cdots, A^{-1} T_{n-1} A y\right\rangle \\
&=\left\langle y, T_{1} y, \cdots, T_{n-1} y\right\rangle \\
&+\left(I^{(n)}-Q\right)\left\langle 0, A^{-1} T_{1} A y, \cdots, A^{-1} T_{n-1} A y\right\rangle,
\end{aligned}
$$

which is clearly in $M$. This completes the proof.

\section{BIBLIOGRAPHY}

1. P. Fillmore and J. P. Williams, On operator ranges, Advances in Math. 7 (1971), 254-281. MR 45 \#2518.

2. C. Foias, Invariant para-closed subspaces, Indiana Univ. Math. J. 21 (1972), 887906.

3. H. Radjavi and P. Rosenthal, On invariant subspaces and reflexive algebras, Amer. J. Math. 91 (1969), 683-692. MR 40 \#4796.

4. J. Wermer, On invariant subspaces of normal operators, Proc. Amer. Math. Soc. 3 (1952), 270-277. MR 14, 55.

Department of Mathematics, University of the Negev, Beer Sheva, Israel 